\title{
Identification of AAV serotypes for lung gene therapy in human embryonic stem cell-derived lung organoids
}

\author{
Helena Meyer-Berg ${ }^{1} \mathbb{D}$, Lucia Zhou Yang ${ }^{2} \mathbb{D}$, María Pilar de Lucas ${ }^{3} \mathbb{D}$, Alberto Zambrano² $\mathbb{D}$, \\ Stephen C. Hyde ${ }^{1}$ (i) and Deborah R. Gill ${ }^{*}$ (1)
}

\begin{abstract}
Gene therapy is being investigated for a range of serious lung diseases, such as cystic fibrosis and emphysema. Recombinant adeno-associated virus (rAAV) is a well-established, safe, viral vector for gene delivery with multiple naturally occurring and artificial serotypes available displaying alternate cell, tissue, and species-specific tropisms. Efficient AAV serotypes for the transduction of the conducting airways have been identified for several species; however, efficient serotypes for human lung parenchyma have not yet been identified. Here, we screened the ability of multiple AAV serotypes to transduce lung bud organoids (LBOs) — a model of human lung parenchyma generated from human embryonic stem cells. Microinjection of LBOs allowed us to model transduction from the luminal surface, similar to dosing via vector inhalation. We identified the naturally occurring rAAV2 and rAAV6 serotypes, along with synthetic rAAV6 variants, as having tropism for the human lung parenchyma. Positive staining of LBOs for surfactant proteins B and C confirmed distal lung identity and suggested the suitability of these vectors for the transduction of alveolar type II cells. Our findings establish LBOs as a new model for pulmonary gene therapy and stress the relevance of LBOs as a viral infection model of the lung parenchyma as relevant in SARSCoV-2 research.
\end{abstract}

Keywords: human embryonic stem cells, stem cell-based tissue model, gene therapy, rAAV, AAV capsids, AAV serotypes, lung organoids, viral infection model, alveolar type II cells

\section{Background}

Recombinant adeno-associated virus (rAAV) is a wellestablished vector for gene delivery, currently in use clinically for gene therapy, with multiple, naturally occurring serotypes and artificial variants facilitating species-specific cell and tissue tropisms [1]. Engineering of new AAV capsids has been the focus of extensive research, but capsids selected in animal models and cancer cell lines often translate poorly to large animal models and humans. Clinical trials of gene therapy for cystic

\footnotetext{
* Correspondence: deborah.gill@ndcls.ox.ac.uk

${ }^{1}$ Gene Medicine Research Group, Nuffield Division of Clinical Laboratory Sciences, Radcliffe Department of Medicine, University of Oxford, Oxford, UK Full list of author information is available at the end of the article
}

fibrosis lung disease using AAV serotype 2 failed to show efficacy [2], and of the many potential reasons for this, an important factor is the lack of serotype screening for airway tropism resulting in poor translation to the human airways [3].

The identification of AAV serotypes for gene delivery to the human lung has focused mainly on the transduction of the human airway epithelium $[4,5]$. The lung parenchyma, however, is the target for treating genetic diseases such as surfactant deficiencies and interstitial lung disease; in particular, alveolar type II (ATII) pneumocytes, which express proteins crucial for surfactant function. 
In this study, we aimed to identify AAV serotypes that permit efficient gene delivery to the human lung parenchyma. We hypothesised that an ideal model for capsid selection should be of human origin and should also offer a polarised cell layer that mimics the tissue surface available to viral vectors, including the distribution of viral entry receptors. We chose a human $3 \mathrm{D}$ cell culture model of the lung as a novel approach for serotype screening-lung bud organoids (LBOs) [6]. The LBOs were generated from human embryonic stem cells (hESC) in this study because their differentiation efficiency is more robust compared to iPSC lines [7] as shown specifically for the generation of progenitor lung cells from iPSC [8]. LBOs exhibit a strong bias towards the generation of lung parenchyma cell types, especially alveolar type II (ATII) pneumocytes [6] and provide a reproducible, in vitro model in which to study human/ viral vector interactions that is substantially more similar to the native tissue environment than traditional, immortalised, submerged cell culture models. The polarised 3D structure of LBOs allows for vector transduction from the luminal surface, mimicking vector administration by inhalation, and thus provides an attractive translational model for diseases of the human parenchyma.

\section{Results}

To generate LBOs, the hESC cell line AND-2 was sequentially differentiated via endoderm and branching induction according to the timeline shown in Fig. 1 [6]. After 59 or 79 days of differentiation in culture, LBOs were microinjected with AAV serotypes 1, 2, 5, 6, 6.2, 6.2FF, 8 , and 9 , or a negative control buffer, to mimic vector delivery to the apical/luminal surface of the lung. Injection of rAAV vectors expressing enhanced green fluorescent protein (EGFP) from the CMV promoter resulted in EGFP-dependent fluorescence in LBOs as early as day 3 post-injection. On day 5 after injection (Fig. 2), high levels of fluorescence directly observed from expressed EGFP ('native' fluorescence) were observed following transduction with rAAV2, rAAV6 and variants rAAV6.2 and rAAV6.2FF ([4, 9], Fig. 2b-e, respectively). Native EGFP fluorescence was much lower in cultures injected with rAAV1 and rAAV8 (Fig. 2f, g), while fluorescence with AAV serotypes 5 and 9 (Fig. 2h, i) was indistinguishable from the mock injection (Fig. 2a). For rAAV6.2, EGFP expression from the CMV promoter was considerably more robust than that achieved with the hCEFI promoter [10], which yielded only low levels of fluorescence (Fig. 2d, j). Analysis of EGFP brightness intensity confirmed that expression levels were highest for rAAV2, rAAV6 and variants rAAV6.2 and rAAV6.2FF which was significantly different from mock injection while rAAV5 and rAAV9 resulted in the lowest levels of brightness (Fig. 2k).

The LBOs were sectioned and stained for ATII cell markers surfactant protein $\mathrm{C}$ (SP-C) and surfactant protein B (SP-B) to confirm distal lung maturity (Fig. 3a, b). Native EGFP-dependent fluorescence was observed alongside positive SP-B immunostaining in LBOs transduced with rAAV6.2-CMV-eGFP (Fig. 3c) compared with non-transduced organoids (Fig. $3 \mathrm{~b}$ ) indicating the suitability of this vector to transduce the human lung parenchyma. To further understand the basis for rAAV transduction, which can depend on both primary glycan receptors and protein co-receptors, we also stained the LBOs for the universal AAV co-receptor (AAVR or KIAA0319L, Fig. 3d), $\alpha$-2,3-linked sialic acid (Fig. 3e, f) and heparan sulphate (Fig. 3g, h). Scattered cells staining positive for $\alpha$-2,3-linked sialic acid were observed

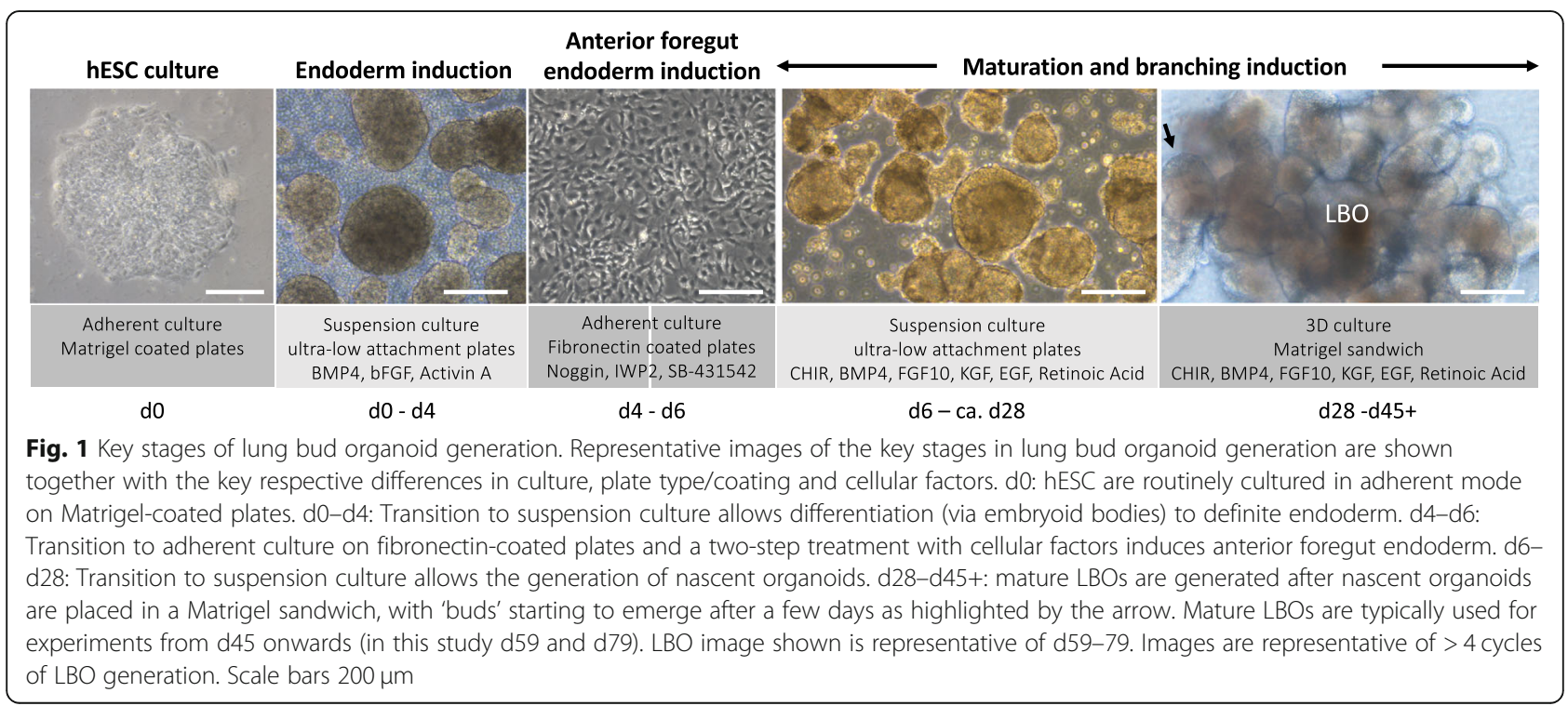



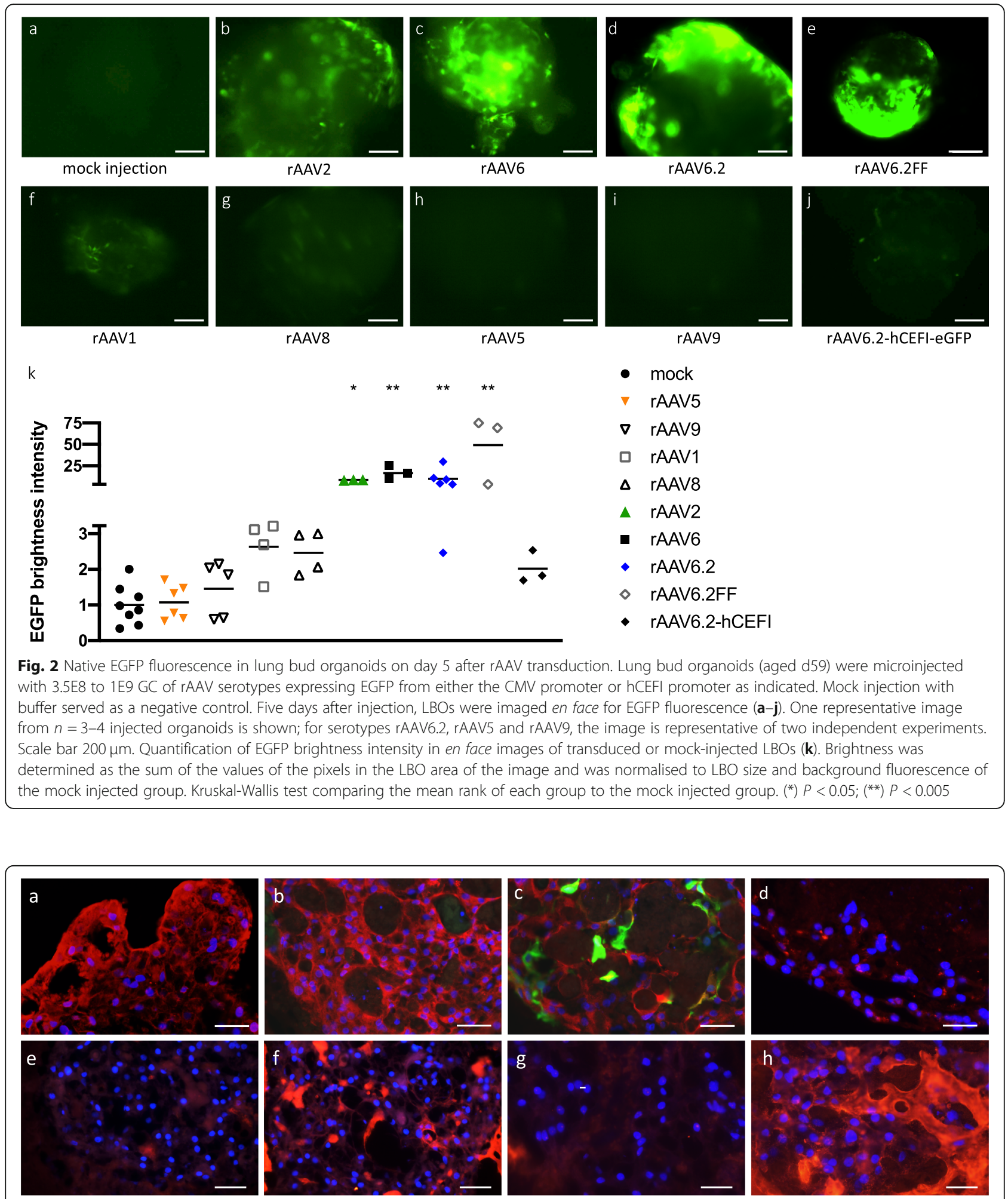

Fig. 3 Immunohistochemistry for markers of alveolar type II cells and AAV entry receptors. Representative images are shown of fixed-frozen sections of LBOs $(n=2-3)$, with nuclei stained with DAPI (blue) and various markers (red), including ATII cell marker SP-C (a), ATII cell marker SP-B $(\mathbf{b}, \mathbf{c})$, universal AAV co-receptor AAVR $(\mathbf{d})$, glycan receptor a-2,3-linked sialic acid $(\mathbf{e}, \mathbf{f})$ and glycan receptor heparan sulphate $(\mathbf{g}, \mathbf{h})$. Native fluorescence is observed following microinjection of AAV6.2-CMV-eGFP (c) compared with negative control LBOs (b). Sections digested with sialidase A (e) and heparinase III (g) to remove glycans are included as negative staining controls. Staining controls for images $\mathbf{a}-\mathbf{d}$ are shown in suppl. Fig. 2. Scale bar $50 \mu \mathrm{m}$ 
(Fig. 3e, f), along with staining of subcellular structures characteristic of AAVR ([11], Fig. 3d) and the 'spotted' staining pattern commonly observed for heparan sulphate ([12], Fig. 3g, h). The observations confirmed the presence of all three rAAV receptor molecules in the LBO cultures.

\section{Discussion}

The development of models of the human lung is important for the investigation of new treatments, but is often challenging when human lung tissue is scarce. Moreover, isolated adult alveolar stem cells quickly dedifferentiate in culture further complicating their use as founder cells for organoid culture. The generation of LBOs from hESC provides a 3D model of the human lung parenchyma, which has been shown to model aspects of embryonic development, RSV infection and genetic diseases such as Hermansky-Pudlak syndrome $[6,13]$. We chose to use this model to identify AAV serotypes with tropism for human lung parenchyma-a crucial step in developing novel viral gene therapies for diseases of the lung.

Microinjection was used to deliver the rAAV vectors to the centre to the LBOs to limit transduction to the apical surface and minimise the opportunity for rAAV transduction via receptors expressed only on the basolateral surface. We showed that reporter EGFP fluorescence was greatest following transduction with AAV serotypes 2, 6 and variants of serotype 6 in d59 organoids, with similar transduction patterns for organoids injected at $\mathrm{d} 79$, indicating the robustness of this model over time (suppl. fig. 1). The AAV5 vector was negative for eGFP expression in this human model (Fig. 2h) although serotype 5 was previously identified as suitable for the transduction of murine lung parenchyma [4]. This highlights the variation in vector tropism observed between species, a well-known challenge in the field of viral in gene therapy, and also more generally a problem for viral infection studies. Staining of the LBOs for the lung parenchymal markers SP-B and SP-C confirmed distal lung identity and suggests the suitability of AAV serotypes 2, 6 and variants of 6 for transduction of ATII cells. Serotypes 6.2 (AAV6 capsid + F129L) and 6.2FF $(\mathrm{AAV6}+\mathrm{F} 129 \mathrm{~L}+\mathrm{Y445F}+\mathrm{Y731F})$ were designed for improved lung transduction $[4,9]$, but in this study, no differences were observed. The hCEFI promoter was investigated for its potential for long-term (months to years) therapeutic transgene expression compared with the strong CMV promoter which is prone to silencing. In a long-term study, native EGFP fluorescence was still detectable with the rAAV6.2 vector on $\mathrm{d} 70$ posttransduction for both the CMV and hCEFI promoters (data not shown). Overall, however, hCEFI expression levels were relatively low in the LBOs (Fig. 2k) suggesting that other promoter options for long-term expression in the human lung parenchyma should be explored, such as the Ubiquitin $\mathrm{C}$ promoter used in the distal lung [14]. Furthermore, staining revealed the presence of the universal AAVR co-receptor, and also $\alpha-2,3-$ linked sialic acid and heparan sulphate, which have been previously observed in resected adult, human lung tissue $[15,16]$. Although the location of these receptors and their subtypes within the human lung and during lung development is not fully understood [12], lectin staining as performed in this study has been reported to intensify in the alveolar region during development from foetal to adult lung [15]. This could lead to improved transduction of adult lung with AAV serotypes dependent on $\alpha$ 2,3-linked sialic acid entry receptors, such as rAAV5, if the glycan receptor rather than a co-receptor was ratelimiting.

The highly efficient transduction of LBOs with AAV serotypes 2 and 6 is consistent with the observed strong positive staining for heparan sulphate (Fig. $3 g$, h), a key entry receptor for these serotypes [1]. These findings indicate that LBO cultures may be a useful model for screening vectors targeting the human parenchyma, particularly in the early (neonatal) stages of lung development, as required in, for example, treatment of congenital surfactant deficiencies. LBOs might also be suitable for the generation of new capsids targeting the human parenchyma via directed evolution and screening of AAV capsids libraries. The LBOs also have the potential to model aspects of respiratory viral infection in the human parenchyma, including infection of ATII cells with MERS-CoV and SARS-CoV-1 and 2 [17]. LBO sections stained positive for the SARS-CoV-2 entry receptors ACE2 and TMPRSS2 (suppl. Fig. 3), supporting our hypothesis that LBOs may be a useful model for SARS$\mathrm{CoV}-2$ research.

In future studies, we anticipate that LBOs can be generated from genome-edited hESCs, giving rise to concomitant morphological and functional phenotypes, that could serve as refined human disease models to facilitate the investigation of therapeutic gene therapy vectors. For example, wildtype LBOs have been shown to recycle SP$B$ from their lumen in a functional assay [6], which could constitute a qualitative read-out of the correction of genetic disorders of surfactant.

In summary, we have established human LBOs as a model to screen for viral vector transduction, identifying serotypes suitable for transduction of the human lung parenchyma.

\section{Methods}

The hESC line AND-2 was sequentially differentiated to LBOs (for details see supplementary methods, Fig. 1 and references $[6,18,19])$. Needles with long continuous 
taper were pulled for LBO microinjection, which was deemed successful when the organoid visibly pulsated during injection (aiming for 3-4 successful injections per site and, depending on the LBO size and number of buds, 2-3 locations; see supplementary methods for detailed microinjection protocol). This corresponded to 3.5E8-1E9 rAAV genome copies (GC) of vector per LBO ( $n=3-4$ LBOs per group). Recombinant AAV vectors were produced by triple transfection in HEK293T cells and purified via iodixanol density gradient centrifugation [20]. Vector purity was tested via SDS-PAGE and titres were determined using quantitative PCR. For immunohistochemistry analysis, LBOs were processed in fixedfrozen sections. Cellular proteins and glycan receptors were stained using primary antibodies to $\alpha$-surfactant protein B (\#sc-133143, Santa Cruz Biotech), $\alpha-$ prosurfactant protein C (\#ab3785, Merck), $\alpha$-KIAA03 19L (AAVR, \#PA5-67257, Invitrogen) and $\alpha$-heparan sulphate, clone F58-10E4 (\#370255, Amsbio), as well as Maackia amurensis Lectin II to detect $\alpha$-2,3-linked sialic acid (\#B-1265, Vectorlabs). As a negative control for staining, glycan receptors were digested using heparinase III or sialidase A and sections processed in parallel.

\section{Supplementary information}

Supplementary information accompanies this paper at https://doi.org/10. 1186/s13287-020-01950-x.

Additional file 1: Supplementary figure 1. Comparison of EGFP brightness intensity in rAAV-dosed d59 vs. d79 organoids. Supplementary figure 2. Negative staining controls (with no primary antibody) for immunohistochemistry on fixed-frozen sections in figure 3. Supplementary figure 3. Immunohistochemistry in LBO sections for entry receptors of SARS-COV-2.

\section{Abbreviations \\ AAV: Adeno-associated virus; AAVR: AAV receptor; ATIl cell: Alveolar type ॥ cell; BMP: Bone morphogenetic protein; CMV: Cytomegalovirus; EGFP: Enhanced green fluorescent protein; FGF: Fibroblast growth factor; GC: Genome copies; hCEFl: Human CMV enhancer coupled to EF1a promoter; hESC: Human embryonic stem cell; KGF: Keratinocyte growth factor; LBO: Lung bud organoid; rAAV: Recombinant AAV; RSV: Respiratory syncytial virus; SARS-CoV: Severe acute respiratory syndrome coronavirus; SP: Surfactant protein}

\section{Acknowledgements}

Not applicable

\section{Authors' contributions}

HMB performed the experiments and analysed and interpreted data. LZY generated organoids and assisted microinjections. MP was instrumental in the development of the organoid microinjection protocol. AZ supervised organoid generation, microinjections and discussed study design. $\mathrm{HMB}, \mathrm{SH}$ and $D G$ designed the study and interpreted the results. $H M B, A Z, S H$ and DG wrote the manuscript in consultation with all authors. The authors read and approved the final manuscript.

\section{Funding}

The material of this study was funded by the Wellcome Trust (110579/Z/15/ Z) and the MRC studentship of HMB.

\section{Availability of data and materials}

Please contact the corresponding author to request datasets used and analysed in this study are available from the corresponding author on reasonable request.

\section{Ethics approval and consent to participate}

The use of the hESC line AND-2 and the experimental procedures of this study were approved by the ISCIII Ethics Committee and the National Committee of Guarantees for the Use and Donation of Human Cells and Tissues (ref. no. 436351 1).

\section{Consent for publication}

Not applicable.

\section{Competing interests}

The authors declare that they have no competing interests.

\section{Author details}

${ }^{1}$ Gene Medicine Research Group, Nuffield Division of Clinical Laboratory Sciences, Radcliffe Department of Medicine, University of Oxford, Oxford, UK. ${ }^{2}$ Department of Biotechnology of Stem Cells and Organoids, Functional Unit for Research into Chronic Diseases, Instituto de Salud Carlos III, Madrid, Spain. ${ }^{3}$ Department of Cellular Biology, Functional Unit for Research into Chronic Diseases, Instituto de Salud Carlos III, Madrid, Spain.

Received: 22 June 2020 Accepted: 24 September 2020

Published online: 23 October 2020

References

1. Li C, Samulski RJ. Engineering adeno-associated virus vectors for gene therapy. Nat Rev Genet. 2020;21(4):255-72.

2. Moss RB, Milla C, Colombo J, Accurso F, Zeitlin PL, Clancy JP, et al. Repeated aerosolized AAV-CFTR for treatment of cystic fibrosis: a randomized placebo-controlled phase 2B trial. Hum Gene Ther. 2007;18(8):726-32.

3. Yan Z, Lei-Butters DC, Liu X, Zhang Y, Zhang L, Luo M, et al. Unique biologic properties of recombinant AAV1 transduction in polarized human airway epithelia. J Biol Chem. 2006;281(40):29684-92.

4. Limberis MP, Vandenberghe LH, Zhang L, Pickles RJ, Wilson JM. Transduction efficiencies of novel AAV vectors in mouse airway epithelium in vivo and human ciliated airway epithelium in vitro. Mol Ther. 2009;17(2): 294-301.

5. Flotte TR, Fischer AC, Goetzmann J, Mueller C, Cebotaru L, Yan Z, et al. Dual reporter comparative indexing of rAAV pseudotyped vectors in chimpanzee airway. Mol Ther. 2010;18(3):594-600.

6. Chen YW, Huang SX, de Carvalho A, Ho SH, Islam MN, Volpi S, et al. A threedimensional model of human lung development and disease from pluripotent stem cells. Nat Cell Biol. 2017;19(5):542-9.

7. Bock C, Kiskinis E, Verstappen G, Gu H, Boulting G, Smith ZD, et al. Reference maps of human ES and iPS cell variation enable high-throughput characterization of pluripotent cell lines. Cell. 2011;144(3):439-52.

8. Huang SX, Green MD, de Carvalho AT, Mumau M, Chen YW, D'Souza SL, et al. The in vitro generation of lung and airway progenitor cells from human pluripotent stem cells. Nat Protoc. 2015;10(3):413-25.

9. van Lieshout LP, Domm JM, Rindler TN, Frost KL, Sorensen DL, Medina SJ, et al. A novel triple-mutant AAV6 capsid induces rapid and potent transgene expression in the muscle and respiratory tract of mice. Mol Ther Methods Clin Dev. 2018;9:323-9.

10. Hyde SC, Pringle IA, Abdullah S, Lawton AE, Davies LA, Varathalingam A, et al. CpG-free plasmids confer reduced inflammation and sustained pulmonary gene expression. Nat Biotechnol. 2008;26(5):549-51.

11. Pillay S, Meyer NL, Puschnik AS, Davulcu O, Diep J, Ishikawa Y, et al. An essential receptor for adeno-associated virus infection. Nature. 2016; 530(7588):108-12.

12. Haeger SM, Yang Y, Schmidt EP. Heparan sulfate in the developing, healthy, and injured lung. Am J Respir Cell Mol Biol. 2016;55(1):5-11.

13. Porotto M, Ferren M, Chen YW, Siu Y, Makhsous N, Rima B, et al. Authentic modeling of human respiratory virus infection in human pluripotent stem cell-derived lung organoids. MBio. 2019;10(3):e00723-19. https://doi.org/10 1128/mBio.00723-19

14. Barnett RC, Lin X, Barravecchia M, Norman RA, de Mesy Bentley KL, Fazal F, et al. Featured article: electroporation-mediated gene delivery of surfactant 
protein B (SP-B) restores expression and improves survival in mouse model of SP-B deficiency. Exp Biol Med (Maywood). 2017;242(13):1345-54.

15. Nicholls JM, Bourne AJ, Chen H, Guan Y, Peiris JS. Sialic acid receptor detection in the human respiratory tract: evidence for widespread distribution of potential binding sites for human and avian influenza viruses. Respir Res. 2007;8:73.

16. Westergren-Thorsson G, Hedstrom U, Nybom A, Tykesson E, Ahrman E, Hornfelt $M$, et al. Increased deposition of glycosaminoglycans and altered structure of heparan sulfate in idiopathic pulmonary fibrosis. Int J Biochem Cell Biol. 2017;83:27-38.

17. Rockx B, Kuiken T, Herfst S, Bestebroer T, Lamers MM, Oude Munnink BB, et al. Comparative pathogenesis of COVID-19, MERS, and SARS in a nonhuman primate model. Science. 2020;368(6494):1012-5.

18. Magro-Lopez E, Guijarro T, Martinez I, Martin-Vicente M, Liste I, Zambrano A. A Two-Dimensional Human Minilung System (Model) for Respiratory Syncytial Virus Infections. Viruses. 2017;9(12):379. Published 2017 Dec 10. https://doi.org/10.3390/v9120379.

19. Magro-Lopez E, Palmer C, Manso J, Liste I, Zambrano A. Effects of lung and airway epithelial maturation cocktail on the structure of lung bud organoids. Stem Cell Res Ther. 2018;9(1):186.

20. de Silva SR, McClements ME, Hankins MW, MacLaren RE. Adeno-associated viral gene therapy for retinal disorders. In: Bo X, Verhaagen J, editors. Gene delivery and therapy for neurological disorders. New York: Springer New York; 2015. p. 203-28.

\section{Publisher's Note}

Springer Nature remains neutral with regard to jurisdictional claims in published maps and institutional affiliations.

Ready to submit your research? Choose BMC and benefit from:

- fast, convenient online submission

- thorough peer review by experienced researchers in your field

- rapid publication on acceptance

- support for research data, including large and complex data types

- gold Open Access which fosters wider collaboration and increased citations

- maximum visibility for your research: over $100 \mathrm{M}$ website views per year

At $\mathrm{BMC}$, research is always in progress.

Learn more biomedcentral.com/submissions 